Document downloaded from:

http://hdl.handle.net/10251/51685

This paper must be cited as:

Benítez López, J.; Rakocevic, V. (2012). Canonical angles and limits of sequences of EP and co-EP matrices. Applied Mathematics and Computation. 218(17):8503-8512. doi:10.1016/j.amc.2012.02.011.

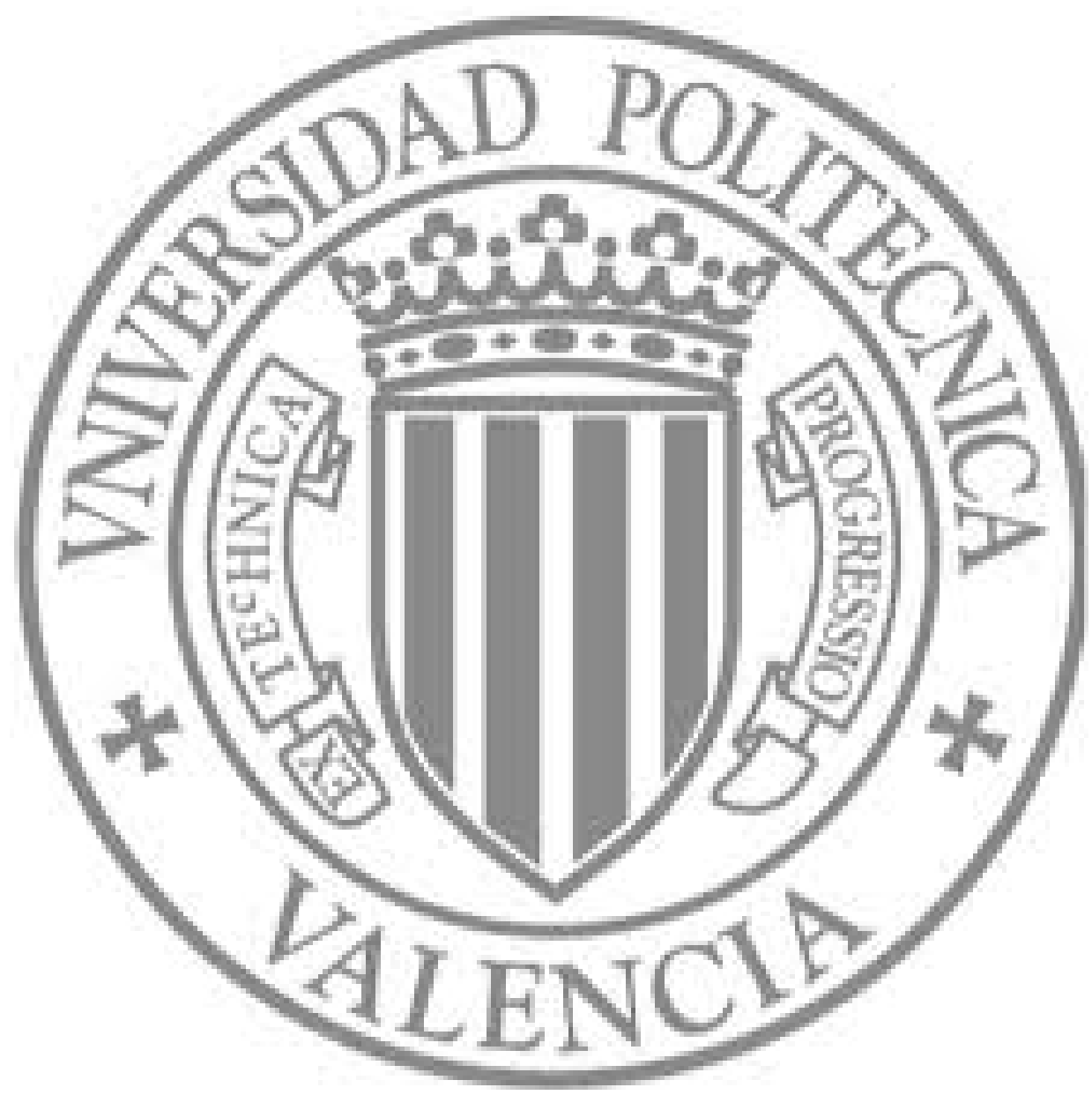

The final publication is available at

http://dx.doi.org/10.1016/j.amc.2012.02.011

Copyright Elsevier 


\title{
Canonical angles and limits of sequences of EP and co-EP matrices
}

\author{
Julio Benítez* $\quad$ Vladimir Rakočević ${ }^{*}$
}

\begin{abstract}
Let $A$ be a square complex matrix. Let $\mathcal{P}$ be one of the following properties: a) $A$ is an EP matrix, $\mathrm{b}$ ) the column space of $A$ is complementary to the column space of $A^{*}$, and c) the orthogonal complement of the column space of $A$ is the column space of $A^{*}$. We study the canonical angles between the column space of $A$ and the column space of $A^{*}$ when $A$ satisfies property $\mathcal{P}$. Also, we research the following problem: Let $\left\{A_{m}\right\}_{m=1}^{\infty}$ be a sequence of matrices satisfying property $\mathcal{P}$ that converges to some matrix $A$. When does $A$ satisfy property $\mathcal{P}$ ?
\end{abstract}

Keywords: Canonical angles of subspaces, EP matrices.

AMS subject classification (2010). 15A09; 15A18.

\section{Introduction, notation, and preliminary results}

The canonical angles (also called principal angles) between two subspaces provide the best available characterization of the relative position of two given subspaces. This concept allows us to characterize or measure, in a natural way, how two subspaces differ, which is the main connection with perturbation theory. In $[1,2,3]$ we can find how these angles were discovered and rediscovered again several times. Computation of canonical angles between subspaces is important in many applications including statistics [4,5], information retrieval [6], and analysis of algorithms [7].

There are many equivalent definitions of the canonical angles (see [8]). For our purposes, the most convenient is the following:

\footnotetext{
* Departamento de Matemática Aplicada, Instituto de Matemática Multidisciplinar, Universidad Politécnica de Valencia, Camino de Vera s/n, 46022, Valencia, Spain. (jbenitez@mat .upv .es).

${ }^{\dagger}$ Departament of Mathematics, Faculty of Science and Mathematics, University of Niš Višegradska, 33, 18000 Niš, Serbia. (vrakoc@bankerinter.net). Supported by Grant No. 144003 of the Ministry of Science, Technology and Development, Republic of Serbia.
} 
Definition 1.1. Let $X$ and $y$ be two nontrivial subspaces of $\mathbb{C}^{n}$ and $r=\min \{\operatorname{dim} X, \operatorname{dim} y\}$. We define the canonical angles $\theta_{1}, \ldots, \theta_{r} \in[0, \pi / 2]$ between $X$ and $y$ by

$$
\cos \theta_{i}=\sigma_{i}\left(P_{x} P_{y}\right), \quad i=1, \ldots, r,
$$

where the real numbers $\sigma_{1}\left(P_{x} P_{y}\right), \cdots, \sigma_{r}\left(P_{x} P_{y}\right) \geq 0$ are the singular values of the matrix $P_{x} P_{y}$, and $P_{\mathcal{S}}$ stands for the orthogonal projector onto the subspace $\mathcal{S} \subset \mathbb{C}^{n}$.

See for example $[8,9,10,11]$ for a deeper insight into this definition and [12] for the simultaneous study of two orthogonal projectors.

Let us remark that the greatest singular value of a matrix $X$ is $\|X\|$, where $\|\cdot\|$ denotes the matrix 2-norm or the matrix norm induced by the Euclidean vector norm. Accordingly to Definition 1.1, the minimal angle between nontrivial subspaces $X$ and $y$ of $\mathbb{C}^{n}$ is defined to be the number $\theta_{x, y} \in[0, \pi / 2]$ for which $\cos \theta_{x, y}=\left\|P_{X} P_{y}\right\|$. It can be proved the following result (see [13, Section 5.15]):

Theorem 1.2. Let $X, Y$ be two nontrivial subspaces of $\mathbb{C}^{n}$. Then $X$ and $y$ are complementary if and only if $P_{X}-P_{y}$ is nonsingular, and in this case

$$
\sin \theta_{x, y}=\frac{1}{\left\|\left(P_{x}-P_{y}\right)^{-1}\right\|}=\frac{1}{\left\|P_{x y}\right\|},
$$

where $P_{x y}$ is the oblique projector onto $X$ along $y$.

Before establishing the aim of this paper, let us fix some standard notations. Let $\mathbb{C}_{m, n}$ denote the set composed of $m \times n$ complex matrices. Throughout this paper we consider the vectors of $\mathbb{C}^{n}$ as columns, thus we shall identify $\mathbb{C}^{n}$ with $\mathbb{C}_{n, 1}$. The symbols $K^{*}, \operatorname{rk}(K), \mathcal{R}(K)$ will denote the conjugate transpose, the rank, and the column space, respectively, of $K \in \mathbb{C}_{m, n}$. Furthermore, $K^{\dagger}$ will stand for the Moore-Penrose inverse of $K \in \mathbb{C}_{m, n}$, i.e., the unique matrix satisfying the four equations

$$
K K^{\dagger} K=K, \quad K^{\dagger} K K^{\dagger}=K^{\dagger}, \quad\left(K K^{\dagger}\right)^{*}=K K^{\dagger}, \quad\left(K^{\dagger} K\right)^{*}=K^{\dagger} K .
$$

It is known that any matrix $K \in \mathbb{C}_{n, m}$ has a Moore-Penrose inverse (and trivially, $K^{\dagger} \in \mathbb{C}_{m, n}$ ). Moreover, it can be easily proved that $K K^{\dagger}$ is the orthogonal projector onto $\mathcal{R}(K)$ and $K^{\dagger} K$ is the orthogonal projector onto $\mathcal{R}\left(K^{*}\right)$. 
A square matrix $A \in \mathbb{C}_{n, n}$ is said to be EP when when $A A^{\dagger}=A^{\dagger} A$. A useful characterization of the EP matrices (the name comes from Equal Projection) is the following (see [14, Chapter 4.4] or [15, Theorem 4.3.1]):

Theorem 1.3. Let $A \in \mathbb{C}_{n, n}$ have rank $r$. The following statements are equivalent:

(i) $A A^{\dagger}=A^{\dagger} A$.

(ii) There exist a unitary matrix $U \in \mathbb{C}_{n, n}$ and a nonsingular matrix $K \in \mathbb{C}_{r, r}$, such that

$$
A=U(K \oplus 0) U^{*}
$$

(iii) $\mathcal{R}(A)=\mathcal{R}\left(A^{*}\right)$.

In some sense, the subset of $\mathbb{C}_{n, n}$ composed of matrices $A$ such that $A A^{\dagger}-A^{\dagger} A$ is nonsingular is complementary to the subset of EP matrices. Following [16] we say that a matrix $A \in \mathbb{C}_{n, n}$ is co-EP when $A A^{\dagger}-A^{\dagger} A$ is nonsingular. Among several characterizations of the co-EP matrices, in [16] the authors proved the following result:

Theorem 1.4. Let $A \in \mathbb{C}_{n, n}$ and $r$ be the rank of $A$. The following statements are equivalent:

(i) $A A^{\dagger}-A^{\dagger} A$ is nonsingular.

(ii) $\mathcal{R}(A) \oplus \mathcal{R}\left(A^{*}\right)=\mathbb{C}_{n, 1}$.

(iii) There exist a unitary matrix $U \in \mathbb{C}_{n, n}$, a nonsingular matrix $M \in \mathbb{C}_{r, r}$, and $\left.\left.\theta_{1}, \ldots, \theta_{r} \in\right] 0, \pi / 2\right]$ such that

$$
A=U\left[\begin{array}{cc}
M C & M S \\
0 & 0
\end{array}\right] U^{*}
$$

where $C=\operatorname{diag}\left(\cos \theta_{1}, \ldots, \cos \theta_{r}\right)$, and $S=\operatorname{diag}\left(\sin \theta_{1}, \ldots, \sin \theta_{r}\right)$.

Obviously, we have $C^{2}+S^{2}=I_{r}$ and $C S=S C$. These two equalities will be used many times in the sequel. Moreover, let us observe that if $A \in \mathbb{C}_{n, n}$ is co-EP matrix, then $n$ must be even and $\operatorname{rk}(A)=n / 2$.

When $\mathcal{R}(A)=\mathcal{R}\left(A^{*}\right)^{\perp}$ (see item (ii) of the previous theorem) holds, then we have the following result [16]: 
Theorem 1.5. Let $A \in \mathbb{C}_{n, n}$ and $r$ be the rank of $A$. The following statements are equivalent:

(i) $\mathcal{R}(A)=\mathcal{R}\left(A^{*}\right)^{\perp}$.

(ii) There exist a unitary matrix $U \in \mathbb{C}_{n, n}$, a nonsingular matrix $M \in \mathbb{C}_{r, r}$ such that

$$
A=U\left[\begin{array}{cc}
0 & M \\
0 & 0
\end{array}\right] U^{*}
$$

(iii) $A A^{\dagger}+A^{\dagger} A=I_{n}$.

(iv) There exists a unitary matrix $U \in \mathbb{C}_{n, n}$ such that $A A^{\dagger}=U\left(I_{r} \oplus 0\right) U^{*}$ and $A^{\dagger} A=U(0 \oplus$ $\left.I_{r}\right) U^{*}$.

We shall say that a matrix $A \in \mathbb{C}_{n, n}$ is co-EP $\mathrm{EP}_{\perp}$ if it satisfies any condition of Theorem 1.5.

The purpose of this paper is twofold: Firstly we study the canonical angles of the EP, co-EP, and co-EP $\perp_{\perp}$ matrices. Lastly we get some results concerning limits of sequences of matrices which are $\mathrm{EP}, \mathrm{co}-\mathrm{EP}, \mathrm{co}-\mathrm{EP}_{\perp}$. These last results have a strong relation with the canonical angles between the column space of some matrices and the column space of their conjugate transposes.

\section{EP, co-EP matrices, and their canonical angles}

If a co-EP matrix $A$ is represented as in (4), then we can know explicitly the canonical angles between $\mathcal{R}(A)$ and $\mathcal{R}\left(A^{*}\right)$.

Theorem 2.1. Let $r \in \mathbb{N}$. If $A \in \mathbb{C}_{2 r, 2 r}$ is represented as

$$
A=U\left[\begin{array}{cc}
M C & M S \\
0 & 0
\end{array}\right] U^{*}
$$

where $U \in \mathbb{C}_{2 r, 2 r}$ is unitary, $M \in \mathbb{C}_{r, r}$ is nonsingular,

$$
C=\operatorname{diag}\left(\cos \theta_{1}, \ldots, \cos \theta_{r}\right), \quad S=\operatorname{diag}\left(\sin \theta_{1}, \ldots, \sin \theta_{r}\right),
$$

and $\theta_{1}, \ldots, \theta_{r} \in[0, \pi / 2]$, then the canonical angles between $\mathcal{R}(A)$ and $\mathcal{R}\left(A^{*}\right)$ are $\theta_{1}, \ldots, \theta_{r}$. 
Proof. First of all, it is easily checked (by using the four conditions in (2)) that

$$
A^{\dagger}=U\left[\begin{array}{ll}
C M^{-1} & 0 \\
S M^{-1} & 0
\end{array}\right] U^{*}
$$

Obvious computations show

$$
A A^{\dagger}=U\left[\begin{array}{cc}
I_{r} & 0 \\
0 & 0
\end{array}\right] U^{*} \quad \text { and } \quad A^{\dagger} A=U\left[\begin{array}{cc}
C^{2} & C S \\
C S & S^{2}
\end{array}\right] U^{*} .
$$

Since $A A^{\dagger}=U\left(I_{r} \oplus 0\right) U^{*}$ we get $\operatorname{dim} \mathcal{R}(A)=\operatorname{rk}\left(A A^{\dagger}\right)=r$ because $A A^{\dagger}$ is the orthogonal projector onto $\mathcal{R}(A)$. Also we have $\operatorname{dim} \mathcal{R}\left(A^{*}\right)=\operatorname{rk}\left(A^{*}\right)=\operatorname{rk}(A)=r$. We shall use (1) in order to find the canonical angles between $\mathcal{R}(A)$ and $\mathcal{R}\left(A^{*}\right)$.

$$
P_{\mathcal{R}(A)} P_{\mathcal{R}\left(A^{*}\right)}=\left(A A^{\dagger}\right)\left(A^{\dagger} A\right)=U\left[\begin{array}{cc}
I_{r} & 0 \\
0 & 0
\end{array}\right]\left[\begin{array}{cc}
C^{2} & C S \\
C S & S^{2}
\end{array}\right] U^{*}=U\left[\begin{array}{cc}
C^{2} & C S \\
0 & 0
\end{array}\right] U^{*} .
$$

It is simple to prove that the matrix $V \in \mathbb{C}_{2 r, 2 r}$ defined by

$$
V=U\left[\begin{array}{cc}
C & -S \\
S & C
\end{array}\right]
$$

is unitary. Since

$$
U\left[\begin{array}{cc}
C^{2} & C S \\
0 & 0
\end{array}\right] U^{*}=U\left[\begin{array}{ll}
C & 0 \\
0 & 0
\end{array}\right]\left[\begin{array}{cc}
C & S \\
-S & C
\end{array}\right] U^{*}=U \operatorname{diag}\left(\cos \theta_{1}, \ldots, \cos \theta_{r}, 0, \ldots, 0\right) V^{*},
$$

the singular value decomposition of $P_{\mathcal{R}(A)} P_{\mathcal{R}\left(A^{*}\right)}$ is

$$
P_{\mathcal{R}(A)} P_{\mathcal{R}\left(A^{*}\right)}=U \operatorname{diag}\left(\cos \theta_{1}, \ldots, \cos \theta_{r}, 0, \ldots, 0\right) V^{*}
$$

Hence the singular values of $P_{\mathcal{R}(A)} P_{\mathcal{R}\left(A^{*}\right)}$ are $\cos \theta_{1}, \ldots, \cos \theta_{r}$. The theorem is proved.

In order to prove some results of this paper, let us permit to write the following factorization valid for all square complex matrix ([17, Cor. 6]).

Lemma 2.2. Any matrix $A \in \mathbb{C}_{n, n}$ of rank $r$ can be represented as

$$
A=U\left[\begin{array}{cc}
\Sigma K & \Sigma L \\
0 & 0
\end{array}\right] U^{*}
$$

where $U \in \mathbb{C}_{n, n}$ is unitary, $\Sigma=\sigma_{1} I_{r_{1}} \oplus \cdots \oplus \sigma_{t} I_{r_{t}}$ is the diagonal matrix of singular values of $A$, $\sigma_{1} \geq \sigma_{2} \geq \cdots \geq \sigma_{t}>0, r_{1}+r_{2}+\cdots+r_{t}=r=\operatorname{rk}(A)$, and $K \in \mathbb{C}_{r, r}, L \in \mathbb{C}_{r, n-r}$ satisfy

$$
K K^{*}+L L^{*}=I_{r}
$$


It is easily seen that if a matrix $A$ is represented as in Lemma 2.2, then

$$
A^{\dagger}=U\left[\begin{array}{ll}
K^{*} \Sigma^{-1} & 0 \\
L^{*} \Sigma^{-1} & 0
\end{array}\right] U^{*}
$$

The following result permits to find the canonical angles between $\mathcal{R}(A)$ and $\mathcal{R}\left(A^{*}\right)$ when $A$ is a matrix represented as in Lemma 2.2.

Theorem 2.3. Let $A \in \mathbb{C}_{n, n}$ be represented as in Lemma 2.2. Then $\theta \in[0, \pi / 2]$ is a canonical angle between $\mathcal{R}(A)$ and $\mathcal{R}\left(A^{*}\right)$ if and only if $\cos \theta$ is a singular value of $K$.

Proof. Let us denote $X=P_{\mathcal{R}(A)} P_{\mathcal{R}\left(A^{*}\right)}$. Since $P_{\mathcal{R}(A)}=A A^{\dagger}$ and $P_{\mathcal{R}\left(A^{*}\right)}=A^{\dagger} A$, using (6) we easily have

$$
\begin{aligned}
X & =\left(A A^{\dagger}\right)\left(A^{\dagger} A\right) \\
& =U\left[\begin{array}{cc}
\Sigma K & \Sigma L \\
0 & 0
\end{array}\right]\left[\begin{array}{ll}
K^{*} \Sigma^{-1} & 0 \\
L^{*} \Sigma^{-1} & 0
\end{array}\right]\left[\begin{array}{cc}
K^{*} \Sigma^{-1} & 0 \\
L^{*} \Sigma^{-1} & 0
\end{array}\right]\left[\begin{array}{cc}
\Sigma K & \Sigma L \\
0 & 0
\end{array}\right] U^{*} \\
& =U\left[\begin{array}{cc}
I_{r} & 0 \\
0 & 0
\end{array}\right]\left[\begin{array}{cc}
K^{*} K & K^{*} L \\
L^{*} K & L^{*} L
\end{array}\right] U^{*} \\
& =U\left[\begin{array}{cc}
K^{*} K & K^{*} L \\
0 & 0
\end{array}\right] U^{*} .
\end{aligned}
$$

It is well known that for every complex square matrix $M$ we have that if $\sigma \geq 0$, then

$\sigma$ is a singular value of $M \Leftrightarrow \sigma^{2}$ is an eigenvalue of $M M^{*} \Leftrightarrow \sigma^{2}$ is an eigenvalue of $M^{*} M$.

Thus, we shall calculate $X X^{*}$. Using (6) leads to

$$
X X^{*}=U\left[\begin{array}{cc}
K^{*} K & 0 \\
0 & 0
\end{array}\right] U^{*} .
$$

Let $\theta \in\left[0, \pi / 2\left[\right.\right.$. The following chain can be easily followed recalling that $X=P_{\mathcal{R}(A)} P_{\mathcal{R}\left(A^{*}\right)}$, Definition 1.1, and $r=\operatorname{rk}(A)$ and therefore, there are exactly $r$ canonical angles between $\mathcal{R}(A)$ and $\mathcal{R}\left(A^{*}\right)$ :

$$
\begin{aligned}
\theta \text { is a canonical angle between } \mathcal{R}(A) \text { and } \mathcal{R}\left(A^{*}\right) & \Leftrightarrow \cos \theta \text { is a singular value of } X \\
& \Leftrightarrow \cos ^{2} \theta \text { is an eigenvalue of } X X^{*} \\
& \Leftrightarrow \cos ^{2} \theta \text { is an eigenvalue of } K^{*} K \\
& \Leftrightarrow \cos \theta \text { is a singular value of } K .
\end{aligned}
$$

The proof is finished. 
The following result links the EP matrices, co-EP matrices, and co-EP $\perp_{\perp}$ matrices with the canonical angles between the column space of these matrices and of their conjugate transposes.

Theorem 2.4. Let $A \in \mathbb{C}_{n, n}$.

(i) $A$ is EP if and only if all the canonical angles between $\mathcal{R}(A)$ and $\mathcal{R}\left(A^{*}\right)$ are zero.

(ii) If $A$ is co-EP, then none of the canonical angles between $\mathcal{R}(A)$ and $\mathcal{R}\left(A^{*}\right)$ are zero.

(iii) If $A$ is co-EP $P_{\perp}$, then all the canonical angles between $\mathcal{R}(A)$ and $\mathcal{R}\left(A^{*}\right)$ are $\pi / 2$.

Proof. It is well known that $P_{\mathcal{R}(A)}=A A^{\dagger}$ and $P_{\mathcal{R}\left(A^{*}\right)}=A^{\dagger} A$. If $r$ is the rank of $A$, then $r=$ $\operatorname{dim} \mathcal{R}(A)=\operatorname{dim} \mathcal{R}\left(A^{*}\right)$.

(i) Suppose that $A$ is EP. Using the representation (3) of $A$ given in Theorem 1.3 we easily get $A A^{\dagger}=A^{\dagger} A=U\left(I_{r} \oplus 0\right) U^{*}$. Hence $P_{\mathcal{R}(A)} P_{\mathcal{R}\left(A^{*}\right)}=U\left(I_{r} \oplus 0\right) U^{*}$. Using (1) proves that all the $r$ canonical angles between $\mathcal{R}(A)$ and $\mathcal{R}\left(A^{*}\right)$ are zero.

Suppose that all the $r$ canonical angles between $\mathcal{R}(A)$ and $\mathcal{R}\left(A^{*}\right)$ are zero. By Theorem 2.3, the scalar 1 is an eigenvalue of $K^{*} K$ with multiplicity $r$; but since $K^{*} K \in \mathbb{C}_{r, r}$ and $K^{*} K$ is diagonalizable, we have $K^{*} K=I_{r}$. This implies that $K^{*}=K^{-1}$ because $K$ is a square matrix. Using (6) we get $L=0$. Representation (5) reduces to $A=U(\Sigma K \oplus 0) U^{*}$. Recall that $\Sigma$ and $K$ are nonsingular, hence item (ii) of Theorem 1.3 yields that $A$ is EP.

(ii) The result follows from Theorem 1.4 and Theorem 2.1.

(iii) If $A$ is co-EP $\perp$, by item (iv) of Theorem 1.5, we get

$$
P_{\mathcal{R}(A)} P_{\mathcal{R}\left(A^{*}\right)}=\left(A A^{\dagger}\right)\left(A^{\dagger} A\right)=0,
$$

hence all the singular values of $P_{\mathcal{R}(A)} P_{\mathcal{R}\left(A^{*}\right)}$ are zero and Definition 1.1 entails that all the $r$ canonical angles between $\mathcal{R}(A)$ and $\mathcal{R}\left(A^{*}\right)$ are $\pi / 2$.

Items (ii) and (iii) of the Theorem 2.4 are not equivalences as the following examples show:

Example 1. Let $B \in \mathbb{C}_{n, n}$ have rank $r$ and let us define $A=B \oplus 0 \in \mathbb{C}_{n+k, n+k}$ with $k>0$. Obviously, we have $\operatorname{rk}(A)=\operatorname{rk}(B)=\operatorname{rk}\left(B^{*}\right)=\operatorname{rk}\left(A^{*}\right)$ and the canonical angles between $\mathcal{R}(A)$ and $\mathcal{R}\left(A^{*}\right)$ are equal to the the canonical angles between $\mathcal{R}(B)$ and $\mathcal{R}\left(B^{*}\right)$. 
If $B$ is co-EP, then none of the $r$ canonical angles between $\mathcal{R}(B)$ and $\mathcal{R}\left(B^{*}\right)$ are zero. Hence, none of the $r$ canonical angles between $\mathcal{R}(A)$ and $\mathcal{R}\left(A^{*}\right)$ are zero. However, $A$ is not co-EP because $A A^{\dagger}-A^{\dagger} A=\left(B B^{\dagger}-B^{\dagger} B\right) \oplus 0$ is a singular matrix.

If $B$ is co-EP $\perp_{\perp}$, all the $r$ canonical angles between $\mathcal{R}(B)$ and $\mathcal{R}\left(B^{*}\right)$ equal to $\pi / 2$. Hence, all the $r$ canonical angles between $\mathcal{R}(A)$ and $\mathcal{R}\left(A^{*}\right)$ are $\pi / 2$. However, $A$ is not co-EP $\perp$ because $A A^{\dagger}+A^{\dagger} A=\left(B B^{\dagger}+B^{\dagger} B\right) \oplus 0=I_{n} \oplus 0 \neq I_{n+k}$ (see item (iii) of Theorem 1.5).

\section{Limits of EP, co-EP matrices and canonical angles}

In this section we shall research the following challenging problem: Let $\mathcal{P}$ be the property of being EP, co-EP, or co-EP $\perp_{\perp}$ and let $\left\{A_{m}\right\}_{m=1}^{\infty} \subset \mathbb{C}_{n, n}$ be a sequence of matrices satisfying property $\mathcal{P}$ that converges to some matrix $A$. When does $A$ satisfy property $\mathcal{P}$ ?

The following simple result is concerned with the above question when the involved matrices are EP. Observe that we must use some kind of continuity of the Moore-Penrose inverse.

Theorem 3.1. Let $\left\{A_{m}\right\}_{m=1}^{\infty}$ be a sequence of EP matrices that converges to $A$. If $A_{m}^{\dagger} \rightarrow A^{\dagger}$, then $A$ is $E P$.

Proof. Since $A_{m}$ is EP for each $m \in \mathbb{N}$ we have $A_{m} A_{m}^{\dagger}=A_{m}^{\dagger} A_{m}$, hence

$$
A A^{\dagger}=\left(\lim _{m} A_{m}\right)\left(\lim _{m} A_{m}^{\dagger}\right)=\lim _{m}\left(A_{m} A_{m}^{\dagger}\right)=\lim _{m}\left(A_{m}^{\dagger} A_{m}\right)=\left(\lim _{m} A_{m}^{\dagger}\right)\left(\lim _{m} A_{m}\right)=A^{\dagger} A .
$$

The proof is finished.

The converse of this result is false as the following example shows:

Example 2. For each natural number, let $A_{m}=1 / m \in \mathbb{C}_{1,1}$. Every $A_{m}$ is evidently EP, the sequence $\left\{A_{m}\right\}_{m=1}^{\infty}$ is convergent, and $\lim _{m} A_{m}=0$ is obviously EP. However, $\left\{A_{m}^{\dagger}\right\}_{m=1}^{\infty}$ does not converge to $0^{\dagger}$.

Contrarily to the usual inverse of a square matrix, it is well known that the Moore-Penrose inverse is not necessarily a continuous function of the elements of the matrix. The following classical theorem gives an equivalent condition for the continuity of the Moore-Penrose inverse of a matrix. 
Theorem $3.2([18,19])$. If $\left\{A_{m}\right\}_{m=1}^{\infty} \subset \mathbb{C}_{n, k}$ is a convergent sequence to $A \in \mathbb{C}_{n, k}$, then

$$
A_{m}^{\dagger} \rightarrow A^{\dagger} \quad \Longleftrightarrow \quad \exists m_{0} \in \mathbb{N}: \operatorname{rk}\left(A_{m}\right)=\operatorname{rk}(A) \forall m \geq m_{0} .
$$

The following examples show that the set of $n \times n$ co-EP matrices and the set of $n \times n$ co$\mathrm{EP}_{\perp}$ matrices are not closed in $\mathbb{C}_{n, n}$.

Example 3. For $m \in \mathbb{N}$, let us define

$$
A_{m}=\left[\begin{array}{cc}
0 & 1 / m \\
0 & 0
\end{array}\right] .
$$

All matrices $A_{m}$ are co-EP $\mathrm{EP}_{\perp}$ and obviously $\lim _{m} A_{m}=0$ is not co-EP $\mathrm{EP}_{\perp}$

Example 4. For $m \in \mathbb{N}$, let us define

$$
B_{m}=\left[\begin{array}{cc}
\cos (1 / m) & \sin (1 / m) \\
0 & 0
\end{array}\right] .
$$

By Theorem 1.4 we have that $B_{m}$ is co-EP for all $m \in \mathbb{N}$. Evidently one has $B_{m} \rightarrow \operatorname{diag}(1,0)$. But $\lim _{m} B_{m}$ is not co-EP (by item (ii) of Theorem 1.3),

Example 3 invites to think about the stability of the rank. This assumption is related with the continuity of the Moore-Penrose inverse.

Let us think about Example 4. Evidently, we have that $\operatorname{dim} \mathcal{R}\left(B_{m}\right)=\operatorname{dim} \mathcal{R}\left(B_{m}^{*}\right)=1$ and $\mathcal{R}\left(B_{m}\right) \oplus \mathcal{R}\left(B_{m}^{*}\right)=\mathbb{C}_{2,1}$ for every $m \in \mathbb{N}$ (this situation is depicted in Figure 1 ) and $1 / m$ is the angle between the lines $\mathcal{R}\left(B_{m}\right)$ and $\mathcal{R}\left(B_{m}^{*}\right)$. When $m \rightarrow \infty$ it is intuitive that these aforementioned lines converge to the same line, say $r$. We have (in a very extremely informal parlance), by denoting $B=\lim _{m} B_{m}=\operatorname{diag}(1,0)$,

$$
\mathcal{R}(B)=\mathcal{R}\left(\lim _{m \rightarrow \infty} B_{m}\right)=\lim _{m \rightarrow \infty} \mathcal{R}\left(B_{m}\right)=r=\lim _{m \rightarrow \infty} \mathcal{R}\left(B_{m}^{*}\right)=\mathcal{R}\left(\lim _{m \rightarrow \infty} B_{m}^{*}\right)=\mathcal{R}\left(B^{*}\right) .
$$

Thus it is impossible that $B$ is a co-EP matrix. In fact, $B$ is an EP matrix.

Let us recall the following fact that we will use in Theorem 3.3 below: If $\left\{X_{m}\right\}_{m=1}^{\infty}$ is a sequence of nonsingular matrices in $\mathbb{C}_{n, n}$ converging to $X$ such that $\left\{X_{m}^{-1}\right\}_{m=1}^{\infty}$ is bounded, then $X$ is nonsingular and $\left\{X_{m}^{-1}\right\}_{m=1}^{\infty}$ converges to $X^{-1}$. In fact, the equality $\left\|X_{k}^{-1}-X_{m}^{-1}\right\|=$ $\left\|X_{k}^{-1}\left(X_{m}-X_{k}\right) X_{m}^{-1}\right\|$ implies that the sequence $\left\{X_{m}^{-1}\right\}_{m=1}^{\infty}$ is a Cauchy sequence, hence convergent, say to $Y$. Now, $I_{n}=X_{m} X_{m}^{-1}$ implies $I_{n}=X Y$.

The following result establishes precise conditions (and a rigorous argument) in order to the limit of co-EP matrices is co-EP. 


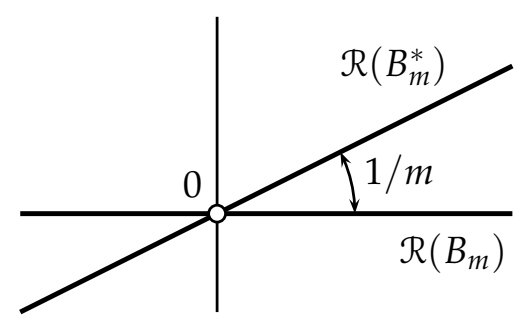

Figure 1: The column space of $B$ is $\operatorname{span}\left\{[1,0]^{*}\right\}$ and the column space of $B_{m}^{*}$ is $\operatorname{span}\left\{[\cos (1 / m), \sin (1 / m)]^{*}\right\}$.

Theorem 3.3. Let $\left\{A_{m}\right\}_{m=1}^{\infty} \subset \mathbb{C}_{n, n}$ be a sequence of co-EP matrices that converges to $A$. Then the following statements are equivalent:

(i) $A_{m}^{\dagger} \rightarrow A^{\dagger}$ and there exists $\theta_{0}>0$ and $m_{0} \in \mathbb{N}$ such that the minimal angle between $\mathcal{R}\left(A_{m}\right)$ and $\mathcal{R}\left(A_{m}^{*}\right)$ is greater than $\theta_{0}$ for all $m \geq m_{0}$.

(ii) $A$ is a co-EP matrix.

Proof. (i) $\Rightarrow$ (ii): Since $A_{m} \rightarrow A$ and $A_{m}^{\dagger} \rightarrow A^{\dagger}$ we get

$$
A_{m} A_{m}^{\dagger}-A_{m}^{\dagger} A_{m} \rightarrow A A^{\dagger}-A^{\dagger} A
$$

On the other hand, matrices $A_{m}$ are co-EP for all $m \in \mathbb{N}$, and thus $A_{m} A_{m}^{\dagger}-A_{m}^{\dagger} A_{m}$ are nonsingular matrices. By using Theorem 1.2

$$
\left\|\left(A_{m} A_{m}^{\dagger}-A_{m}^{\dagger} A_{m}\right)^{-1}\right\|_{2}=\frac{1}{\sin \theta_{\mathcal{R}\left(A_{m}\right), \mathcal{R}\left(A_{m}^{*}\right)}} \leq \frac{1}{\sin \theta_{0}}
$$

holds for $m \geq m_{0}$. Therefore, the sequence $\left\{\left(A_{m} A_{m}^{\dagger}-A_{m}^{\dagger} A_{m}\right)^{-1}\right\}_{m=1}^{\infty}$ is bounded. The use of (7) ensures now that $A A^{\dagger}-A^{\dagger} A$ in nonsingular, or in another words, $A A^{\dagger}-A^{\dagger} A$ is co-EP.

(ii) $\Rightarrow$ (i): Since $A$ and $A_{m}$ are co-EP matrices, we get $\operatorname{rk}\left(A_{m}\right)=\operatorname{rk}(A)=n / 2$ for all $m \in \mathbb{N}$. By Theorem 3.2 we get $A_{m}^{\dagger} \rightarrow A^{\dagger}$, hence $A_{m} A_{m}^{\dagger}-A_{m}^{\dagger} A_{m} \rightarrow A A^{\dagger}-A^{\dagger} A$. Since $A_{m} A_{m}^{\dagger}-A_{m}^{\dagger} A_{m}$ and $A A^{\dagger}-A^{\dagger} A$ are nonsingular matrices, the continuity of the inverse permits to affirm that

$$
\lim _{m \rightarrow \infty}\left(A_{m} A_{m}^{\dagger}-A_{m}^{\dagger} A_{m}\right)^{-1}=\left(A A^{\dagger}-A^{\dagger} A\right)^{-1},
$$

in particular, the sequence $\left\{\left\|\left(A_{m} A_{m}^{\dagger}-A_{m}^{\dagger} A_{m}\right)^{-1}\right\|\right\}_{m=1}^{\infty}$ is bounded, say by $K$. Using Theorem 1.2 we get

$$
\sin \theta_{\mathcal{R}\left(A_{m}\right), \mathcal{R}\left(A_{m}^{*}\right)}=\frac{1}{\left\|\left(A_{m} A_{m}^{\dagger}-A_{m}^{\dagger} A_{m}\right)^{-1}\right\|} \geq \frac{1}{K}
$$


The proof is completed.

A related result holds when the involved matrices are co-EP : $^{\circ}$

Theorem 3.4. Let $\left\{A_{m}\right\}_{m=1}^{\infty} \subset \mathbb{C}_{n, n}$ be a sequence of co-EP $\perp$ matrices that converges to $A$. Then the following statements are equivalent:

(i) $A_{m}^{\dagger} \rightarrow A^{\dagger}$.

(ii) $A$ is co-EP $\perp$.

Proof. (i) $\Rightarrow$ (ii): By item (iv) of Theorem 1.5 we get $A_{m} A_{m}^{\dagger}+A_{m}^{\dagger} A_{m}=I_{n}$ for each $m \in \mathbb{N}$. Since $A_{m} \rightarrow A$ and $A_{m}^{\dagger} \rightarrow A^{\dagger}$, the results follows.

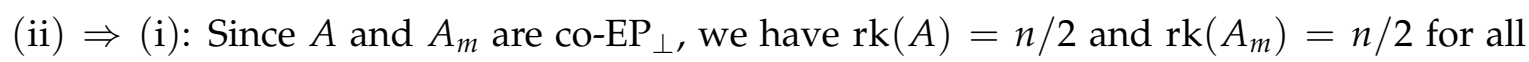
$m \in \mathbb{N}$. Theorem 3.2 ends the proof.

The following simple lemmas will be used in the sequel.

Lemma 3.5. Let $K \in \mathbb{C}_{r, r}$ and $L \in \mathbb{C}_{r, n-r}$ be such $K K^{*}+L L^{*}=I_{r}$, then $\|K\| \leq 1,\|L\| \leq 1$.

Proof. Let us define

$$
A=\left[\begin{array}{ll}
K & L \\
0 & 0
\end{array}\right]
$$

It is easily checked that $A A^{*}=I_{r} \oplus 0$. Hence $1=\left\|I_{r} \oplus 0\right\|=\left\|A A^{*}\right\|=\|A\|^{2}$, and thus $\max \{\|K\|,\|L\|\} \leq\|A\|=1$ (the norm of a submatrix cannot be greater than the norm of a whole matrix, see e.g. [20, Lemma 2]).

Lemma 3.6. The set $\left\{(A, B) \in \mathbb{C}_{r, r} \times \mathbb{C}_{r, n-r}: A A^{*}+B B^{*}=I_{r}\right\}$ is a compact subset of $\mathbb{C}_{r, r} \times \mathbb{C}_{r, n-r}$. Proof. Let us prove that $\mathcal{H}=\left\{(A, B) \in \mathbb{C}_{r, r} \times \mathbb{C}_{r, n-r}: A A^{*}+B B^{*}=I_{r}\right\}$ is a compact subset of $\mathbb{C}_{r, r} \times \mathbb{C}_{r, n-r}$. By Lemma 3.5 we get that $\mathcal{H}$ is bounded. Now, let us consider the continuous map $\Phi: \mathbb{C}_{r, r} \times \mathbb{C}_{r, n-r} \rightarrow \mathbb{C}_{r, r}$ given by $\Phi(X, Y)=X X^{*}+Y Y^{*}$. Since $\mathcal{H}=\Phi^{-1}\left(I_{r}\right)$ we get that $\mathcal{H}$ is closed in $\mathbb{C}_{r, r} \times \mathbb{C}_{r, n-r}$. This finishes the proof.

Let us recall that the set composed of unitary $n \times n$ matrices is compact in $\mathbb{C}_{n, n}$. Also, let us recall that any sequence in a compact set has a convergent subsequence.

The following result concerns EP matrices. 
Theorem 3.7. Let $\left\{A_{m}\right\}_{m=1}^{\infty} \subset \mathbb{C}_{n, n}$ be a convergent sequence of matrices such that $A_{m} \rightarrow A$ and $A_{m}^{\dagger} \rightarrow A^{\dagger}$. Let $\phi_{m}$ be the greatest canonical angle between $\mathcal{R}\left(A_{m}\right)$ and $\mathcal{R}\left(A_{m}^{*}\right)$. The following statements are equivalent:

(i) $\phi_{m} \rightarrow 0$.

(ii) $A$ is EP.

Proof. By Theorem 3.2 there exists $m_{0} \in \mathbb{N}$ such that $\operatorname{rk}\left(A_{m}\right)=\operatorname{rk}(A)$ for $m \geq m_{0}$. Let us denote $r=\operatorname{rk}(A)$. By Lemma 2.2, we can write for $m \geq m_{0}$

$$
A_{m}=U_{m}\left[\begin{array}{cc}
\Sigma_{m} K_{m} & \Sigma_{m} L_{m} \\
0 & 0
\end{array}\right] U_{m}^{*}
$$

where $\Sigma_{m}$ are diagonal and invertible, $U_{m}$ unitary, and

$$
K_{m} K_{m}^{*}+L_{m} L_{m}^{*}=I_{r}
$$

Also, we have

$$
A_{m}^{\dagger}=U_{m}\left[\begin{array}{ll}
K_{m}^{*} \Sigma_{m}^{-1} & 0 \\
L_{m}^{*} \Sigma_{m}^{-1} & 0
\end{array}\right] U_{m}^{*}
$$

By passing to a subsequence, we can suppose that $\left\{U_{m}\right\}_{m=1}^{\infty}$ is convergent to the unitary matrix $U$. Since the sequences $\left\{A_{m}\right\}_{m=1}^{\infty},\left\{A_{m}^{\dagger}\right\}_{m=1}^{\infty}$, and $\left\{U_{m}\right\}_{m=1}^{\infty}$, are convergent, from representations (8) and (9) we get that the following four sequences $\left\{\Sigma_{m} K_{m}\right\}_{m=1}^{\infty},\left\{\Sigma_{m} L_{m}\right\}_{m=1}^{\infty}$, $\left\{K_{m}^{*} \Sigma_{m}^{-1}\right\}_{m=1}^{\infty}$, and $\left\{L_{m}^{*} \Sigma_{m}^{-1}\right\}_{m=1}^{\infty}$ are convergent, say to $X, Y, Z$, and $T$, respectively. Since we have $A_{m} \rightarrow A$ and $A_{m}^{\dagger} \rightarrow A^{\dagger}$ we get

$$
A=U\left[\begin{array}{cc}
X & Y \\
0 & 0
\end{array}\right] U^{*}, \quad A^{\dagger}=U\left[\begin{array}{cc}
Z & 0 \\
T & 0
\end{array}\right] U^{*}
$$

We will prove that the sequences $\Sigma_{m}$ and $\Sigma_{m}^{-1}$ are bounded: In fact by Lemma 3.5 and recalling that $\left\{\Sigma_{m} L_{m}\right\}_{m=1}^{\infty},\left\{K_{m}^{*} \Sigma_{m}^{-1}\right\}_{m=1}^{\infty}$, and $\left\{L_{m}^{*} \Sigma_{m}^{-1}\right\}_{m=1}^{\infty}$ are convergent,

$$
\left\|\Sigma_{m}\right\|=\left\|\Sigma_{m} K_{m} K_{m}^{*}+\Sigma_{m} L_{m} L_{m}^{*}\right\| \leq\left\|\Sigma_{m} K_{m}\right\|+\left\|\Sigma_{m} L_{m}\right\|
$$

and

$$
\left\|\Sigma_{m}^{-1}\right\|=\left\|K_{m} K_{m}^{*} \Sigma_{m}^{-1}+L_{m} L_{m}^{*} \Sigma_{m}^{-1}\right\| \leq\left\|K_{m}^{*} \Sigma_{m}^{-1}\right\|+\left\|L_{m}^{*} \Sigma_{m}^{-1}\right\| .
$$


Thus, denoting by $\theta_{1}, \ldots, \theta_{r}$ the canonical angles between $\mathcal{R}(A)$ and $\mathcal{R}\left(A^{*}\right)$, from Theorem 2.3 and (9), we have

$$
\begin{aligned}
\phi_{m} & \rightarrow 0 \Leftrightarrow \cos ^{2} \theta_{i} \rightarrow 1 \text { for all } i=1, \ldots, r \\
& \Leftrightarrow K_{m} K_{m}^{*} \rightarrow I_{r} \Leftrightarrow L_{m} L_{m}^{*} \rightarrow 0 \Leftrightarrow L_{m} \rightarrow 0 \Leftrightarrow \Sigma_{m} L_{m} \rightarrow 0 \Leftrightarrow Y=0 .
\end{aligned}
$$

Therefore, to demonstrate this theorem, it is enough to prove that $Y=0$ if and only if $A$ is EP.

(i) $\Rightarrow$ (ii): If $Y=0$, then from

$$
I_{r}=\Sigma_{m} K_{m} K_{m}^{*} \Sigma_{m}^{-1}+\Sigma_{m} L_{m} L_{m}^{*} \Sigma_{m}^{-1} \rightarrow X Z+0 \cdot T=X Z,
$$

we deduce $X Z=I_{r}$, hence $X$ is nonsingular and the first representation in (10) and Theorem 1.3 yields that $A$ is EP.

(ii) $\Rightarrow$ (i): If $A$ is EP, then $A A^{\dagger}=A^{\dagger} A$. Now, equality (10) implies

$$
X Z+Y T=Z X, \quad 0=Z Y, \quad 0=T X, \quad 0=T Y
$$

Moreover, since $I_{r}=\Sigma_{m} K_{m} K_{m}^{*} \Sigma_{m}^{-1}+\Sigma_{m} L_{m} L_{m}^{*} \Sigma_{m}^{-1}$, making $k \rightarrow \infty$ we get $I_{r}=X Z+Y T$. Thus, the first equality of (12) leads to $Z X=I_{r}$. Let us remark that both matrices $Z$ and $X$ are square, thus $X$ and $Z$ are nonsingular and from the second equality of (12) we get $Y=0$. The proof is finished

Let $\left\{A_{m}\right\}_{m=1}^{\infty} \subset \mathbb{C}_{n, n}$ be a convergent sequence to $A$. Let $\phi_{m}$ the greatest canonical between $\mathcal{R}\left(A_{m}\right)$ and $\mathcal{R}\left(A_{m}^{*}\right)$. If $\phi_{m} \rightarrow 0$, it is reasonable to think that the canonical angles between $\mathcal{R}(A)$ and $\mathcal{R}\left(A^{*}\right)$ are 0 , or according to Theorem 2.4, $A$ is EP. But, the following example shows that this intuition is false:

Example 5. Let $\left\{a_{m}\right\}_{m=1}^{\infty}$ be a sequence of numbers in $] 0,1\left[\right.$ converging to 1 , and define $b_{m}=$ $\sqrt{1-a_{m}^{2}}$

$$
K_{m}=\left[\begin{array}{cc}
0 & a_{m} \\
a_{m} & 0
\end{array}\right], \quad L_{m}=\left[\begin{array}{cc}
0 & b_{m} \\
b_{m} & 0
\end{array}\right], \quad \Sigma_{m}=\left[\begin{array}{cc}
1 & 0 \\
0 & 1 / m
\end{array}\right], \quad A_{m}=\left[\begin{array}{cc}
\Sigma_{m} K_{m} & \Sigma_{m} L_{m} \\
0 & 0
\end{array}\right] .
$$

It is straightforward to prove $K_{m} K_{m}^{*}=a_{m}^{2} I_{2}$, and thus $K_{m}$ has only one singular value, namely $a_{m}$. Moreover, $L_{m} L_{m}^{*}=b_{m}^{2} I_{2}$, and therefore $K_{m} K_{m}^{*}+L_{m} L_{m}^{*}=I_{2}$ holds. By Theorem 2.3, the angle $\arccos \left(a_{m}\right)$ is the unique canonical angle between $\mathcal{R}\left(A_{m}\right)$ and $\mathcal{R}\left(A_{m}^{*}\right)$. Using the notation 
of Theorem 3.7, one has $\phi_{m}=\arccos \left(a_{m}\right)$. Since $a_{m} \rightarrow 1$ we get $\phi_{m} \rightarrow 0$. However, the sequence $\left\{A_{m}\right\}_{m=1}^{\infty}$ converges to

$$
A=\left[\begin{array}{cc}
\Sigma K & 0 \\
0 & 0
\end{array}\right], \quad \text { where } K=\left[\begin{array}{ll}
0 & 1 \\
1 & 0
\end{array}\right] \text { and } \Sigma=\left[\begin{array}{ll}
1 & 0 \\
0 & 0
\end{array}\right] .
$$

However, matrix $A$ is not EP because if $A$ were EP, then matrix $\Sigma K$ would be also EP; but since

$$
\Sigma K=\left[\begin{array}{ll}
1 & 0 \\
0 & 0
\end{array}\right]\left[\begin{array}{ll}
0 & 1 \\
1 & 0
\end{array}\right]=\left[\begin{array}{ll}
0 & 1 \\
0 & 0
\end{array}\right]
$$

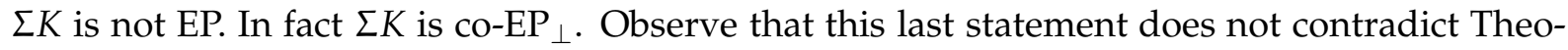
rem 2.4.

The following result does not require the continuity of the Moore-Penrose inverse. Let us remark that any Hermitian matrix is EP.

Theorem 3.8. Let $\left\{A_{m}\right\}_{m=1}^{\infty} \subset \mathbb{C}_{n, n}$ be a convergent sequence to $A$. Let $\phi_{m}$ the greatest canonical between $\mathcal{R}\left(A_{m}\right)$ and $\mathcal{R}\left(A_{m}^{*}\right)$. If $\phi_{m} \rightarrow 0$ and $P_{\mathcal{R}\left(A_{m}\right)}\left(A_{m}-A_{m}^{*}\right) P_{\mathcal{R}\left(A_{m}\right)} \rightarrow 0$, then $A$ is Hermitian.

Proof. Let $r_{m}=\operatorname{rk}\left(A_{m}\right)$. Since $r_{m} \in\{1, \cdots, n\}$ for all $m \in \mathbb{N}$, by passing to a subsequence, we can suppose that $\left\{r_{m}\right\}_{m=1}^{\infty}$ is constant, say to $r$. By Lemma 2.2, for every $m \in \mathbb{N}$, there exist a unitary matrix $U_{m} \in \mathbb{C}_{n, n}$, a diagonal and positive matrix $\Sigma_{m} \in \mathbb{C}_{r, r}$, and $\left(K_{m}, L_{m}\right) \in$ $\mathbb{C}_{r, r} \times \mathbb{C}_{r, n-r}$ such that

$$
A_{m}=U_{m}\left[\begin{array}{cc}
\Sigma_{m} K_{m} & \Sigma_{m} L_{m} \\
0 & 0
\end{array}\right] U_{m}^{*}
$$

and

$$
K_{m} K_{m}^{*}+L_{m} L_{m}^{*}=I_{r}
$$

Since the subset of unitary matrices is compact and by Lemma 3.6, by passing again to another subsequence, we can suppose that $\left\{U_{m}\right\}_{m=1}^{\infty},\left\{K_{m}\right\}_{m=1}^{\infty}$, and $\left\{L_{m}\right\}_{m=1}^{\infty}$ are convergent sequences to, respectively, $U, K, L$. These matrices satisfy

$$
U U^{*}=U^{*} U=I_{n}, \quad K K^{*}+L L^{*}=I_{r} .
$$

By the proof of Theorem 3.7 (see (11)), since $\phi_{m} \rightarrow 0$ we get $L_{m} \rightarrow 0$ i.e., $L=0$. From the second equality of (14) we get $K K^{*}=I_{r}$. Observe that

$$
\left[\begin{array}{cc}
\Sigma_{m} K_{m} & \Sigma_{m} L_{m} \\
0 & 0
\end{array}\right]=U_{m}^{*} A_{m} U_{m} \rightarrow U^{*} A U,
$$


hence $\left\{\Sigma_{m} K_{m}\right\}_{m=1}^{\infty}$ and $\left\{\Sigma_{m} L_{m}\right\}_{m=1}^{\infty}$ are sequences that converge to $X$ and $Y$, respectively. Moreover we get

$$
\left[\begin{array}{cc}
X & Y \\
0 & 0
\end{array}\right]=U^{*} A U
$$

Since $K K^{*}=I_{r}$, in particular $K$ is nonsingular (recall that $K$ is a square matrix) and for big enough $m$, one has that $K_{m}$ is nonsingular. Hence, the continuity of the inverse in the subset composed of nonsingular matrices permits assure that $K_{m}^{-1} \rightarrow K^{-1}$. Now, for big enough $m$, we have

$$
\Sigma_{m}=\Sigma_{m} K_{m} K_{m}^{-1} \rightarrow X K^{-1}
$$

and $\Sigma_{m} L_{m} \rightarrow X K^{-1} 0=0$. Since $Y=\lim _{m} \Sigma_{m} L_{m}$, by the uniqueness of the limit, we get $Y=0$. Thus, we get

$$
A=U\left[\begin{array}{cc}
X & 0 \\
0 & 0
\end{array}\right] U^{*}, \quad A^{\dagger}=U\left[\begin{array}{cc}
X^{\dagger} & 0 \\
0 & 0
\end{array}\right] U^{*}
$$

Denote $\Sigma=X K^{-1}$. Evidently, we have $X=\Sigma K$. From (15), matrix $\Sigma$ is a limit of diagonal and real matrices, hence $\Sigma$ is also diagonal and real. In particular we get $\Sigma^{*}=\Sigma$.

Using representation (13) we have

$$
P_{\mathcal{R}\left(A_{m}\right)}\left(A_{m}-A_{m}^{*}\right) P_{\mathcal{R}\left(A_{m}\right)}=U_{m}\left[\begin{array}{cc}
\Sigma_{m} K_{m}-K_{m}^{*} \Sigma_{m}^{*} & 0 \\
0 & 0
\end{array}\right] U_{m}^{*} \rightarrow U\left[\begin{array}{cc}
\Sigma K-K^{*} \Sigma & 0 \\
0 & 0
\end{array}\right] U^{*}
$$

From $P_{\mathcal{R}\left(A_{m}\right)}\left(A_{m}-A_{m}^{*}\right) P_{\mathcal{R}\left(A_{m}\right)} \rightarrow 0$ we get $\Sigma K=K^{*} \Sigma$. Now let us prove that $X$ is Hermitian:

$$
X^{*}=(\Sigma K)^{*}=K^{*} \Sigma^{*}=K^{*} \Sigma=\Sigma K=X .
$$

The representation of $A$ given in (16) proves that $A$ is Hermitian.

Example 6. The following example shows that we cannot remove the condition

$$
\lim _{m \rightarrow \infty} P_{\mathcal{R}\left(A_{m}\right)}\left(A_{m}-A_{m}^{*}\right) P_{\mathcal{R}\left(A_{m}\right)}=0
$$

in Theorem 3.8. Let

$$
A_{m}=U\left[\begin{array}{cc}
1 & 0 \\
0 & 1 / m
\end{array}\right], \quad A=U\left[\begin{array}{ll}
1 & 0 \\
0 & 0
\end{array}\right], \quad U=\left[\begin{array}{cc}
1 / \sqrt{2} & 1 / \sqrt{2} \\
-1 / \sqrt{2} & 1 / \sqrt{2}
\end{array}\right] .
$$

Note that $U$ is unitary. Since $A_{m}$ is nonsingular, the maximal angle between $\mathcal{R}\left(A_{m}\right)$ and $\mathcal{R}\left(A_{m}^{*}\right)$ is zero for all $m \in \mathbb{N}$. Clearly $A_{m} \rightarrow A$. Now, let us compute the maximal angle between $\mathcal{R}(A)$ and $\mathcal{R}\left(A^{*}\right)$ : Since

$$
P_{\mathcal{R}(A)} P_{\mathcal{R}\left(A^{*}\right)}=A A^{\dagger} A^{\dagger} A=\left[\begin{array}{cc}
1 / 2 & 0 \\
-1 / 2 & 0
\end{array}\right]
$$


Definition 1.1 shows that if $\phi$ the maximal canonical angle between $\mathcal{R}(A)$ and $\mathcal{R}\left(A^{*}\right)$ (in fact is the only canonical angle between $\mathcal{R}(A)$ and $\left.\mathcal{R}\left(A^{*}\right)\right)$, then $\cos ^{2} \phi=1 / 2$, i.e., $\phi=\pi / 4$.

Since $A_{m}$ is nonsingular for each $m$, we have that $P_{\mathcal{R}\left(A_{m}\right)}=P_{\mathcal{R}\left(A_{m}\right)}=I_{2}$. Thus

$P_{\mathcal{R}\left(A_{m}\right)}\left(A_{m}-A_{m}^{*}\right) P_{\mathcal{R}\left(A_{m}\right)}=U\left[\begin{array}{cc}1 & 0 \\ 0 & 1 / m\end{array}\right]-\left[\begin{array}{cc}1 & 0 \\ 0 & 1 / m\end{array}\right] U^{*} \rightarrow U\left[\begin{array}{ll}1 & 0 \\ 0 & 0\end{array}\right]-\left[\begin{array}{ll}1 & 0 \\ 0 & 0\end{array}\right] U^{*} \neq 0$.

Example 7. Let $\left\{A_{m}\right\}_{m=1}^{\infty}$ be a sequence of matrices converging to $A$. The following conditions

(a) $\lim _{m \rightarrow \infty} A_{m}^{\dagger}=A^{\dagger}$.

(b) $\lim _{m \rightarrow \infty} P_{\mathcal{R}\left(A_{m}\right)}\left(A_{m}-A_{m}^{*}\right) P_{\mathcal{R}\left(A_{m}\right)}=0$.

are independent, as the following examples show.

Let $A_{m}=1 / m \in \mathbb{C}_{1,1}$ and $A=0$. Obviously, the sequence $\left\{A_{m}\right\}_{m=1}^{\infty}$ satisfy condition (b) and does not satisfy condition (a).

Now let $B \in \mathbb{C}_{n, n}$ be any nonsingular and non Hermitian matrix. For any $m \in \mathbb{N}$ let us define $B_{m}=B$. The sequence $\left\{B_{m}\right\}_{m=1}^{\infty}$ satisfy condition (a). However, this sequence does not satisfy condition (b): Observe that $P_{\mathcal{R}\left(B_{m}\right)}=I_{n}$ since $B_{m}$ is nonsingular, therefore, $\lim _{m \rightarrow \infty} P_{\mathcal{R}\left(B_{m}\right)}\left(B_{m}-B_{m}^{*}\right) P_{\mathcal{R}\left(B_{m}\right)}=B-B^{*} \neq 0$.

Acknowledgements: The authors wish to thank the referee for his/her careful review and comments which improved the quality of this article.

\section{References}

[1] C. Davis, W.M. Kahan, The rotation of eigenvectors by a perturbation. III, SIAM J. Numer. Anal. 7 (1970), 1-46.

[2] C.C. Paige, M.Wei, History and generality of the CS decomposition, Linear Algebra Appl., 208-209 (1994) 303-326.

[3] I.B. Risteski, K.G. Trencevski, Principal Values and Principal Subspaces of Two Subspaces of Vector Spaces with Inner Product, Beiträge Algebra Geom., 42 (2001), 289-300.

[4] J. Dauxois, G. M. Nkiet, Canonical analysis of two Euclidean subspaces and its applications, Linear Algebra Appl., 264 (1997), 355-388. 
[5] H. Hotelling, Relation between two sets of variables, Biometrica, 28 (1936),322-377.

[6] E.R. Jessup, M.W. Berry, Z. Drmac, Matrices, vector spaces, and information retrieval, SIAM Review, 41 (1999), 335-362.

[7] G.W. Stewart. Matrix Algorithms Volume II: Eigensystems. SIAM, Philadelphia, PA, (2001).

[8] A. Galantai, Cs.J. Hegedus, Jordan's principal angles in complex vector spaces, Num. Lin. Alg. Appl., 13 (2006), 589-598.

[9] G. H. Golub and C. F. Van Loan, Matrix Computations, third ed., Johns Hopkins Studies in the Mathematical Sciences, Johns Hopkins University Press, Baltimore, MD (1996).

[10] V. Rakočević, H. K. Wimmer, A variational characterization of canonical angles between subspaces, J. Geom., 78 (2003), 122-124.

[11] H.K. Wimmer, Canonical angles of unitary spaces and perturbations of direct complements, Linear Algebra Appl., 287 (1999), 373-379.

[12] J. Benítez, V. Rakočević, Applications of CS decomposition in linear combinations of two orthogonal projectors, Appl. Math. Comput., 203 (2008), 761-769.

[13] C.D. Meyer, Matrix analysis and applied linear algebra, SIAM, Philadelphia, PA, (2000).

[14] A. Ben-Israel, T. N. E. Greville, Generalized Inverses, Springer-Verlag, New York, 2003.

[15] S.L. Campbell, C.D. Meyer, Jr, Generalized inverses of Linear Transformations. Pitman, London, 1979.

[16] J. Benítez, V. Rakočević, Matrices $A$ such that $A A^{\dagger}-A^{\dagger} A$ are nonsingular, Appl. Math. Comput. 217 (2010) 3493-3503.

[17] R.E. Hartwig, K. Spindelböck, Matrices for which $A^{*}$ and $A^{\dagger}$ commute, Linear Multilinear Algebra, 14 (1984), 241-256.

[18] R. Penrose, A Generalized Inverse for Matrices, Proc. Cambridge Phil. Soc., 51 (1955) 406413. 
[19] G.W. Stewart, On the continuity of the generalized inverse, SIAM J. Appl. Math., 17 (1969), 33-45.

[20] O.M. Baksalary, J. Benítez, On linear combinations of two commuting hypergeneralized projectors, Comput. Math. Appl., 56 (2008), 2481-2489. 